\title{
Proyecto económico para la fabricación y comercialización de una bebida energizante de taxo endulzada con estevia
}

\author{
Economic project for the manufacture and commercialization of an \\ energizing taxo drink sweetened with estevia
}
María Belén Bravo Avalos. ${ }^{1}$, Luis Antonio Velasco Matveev. ${ }^{2}$ \& Santiago Nicolás Aguiar Novillo. ${ }^{3}$

\begin{abstract}
.
DOI: https://doi.org/10.33262/concienciadigital.v4i3.1.1854

Introduction. Currently the market for energy drinks has increased, this research aims to analyze the capacity of the market in the city of Riobamba to accept a drink made with a product from the area: taxo, a fruit that provides vitamins and minerals, has a very characteristic smell and pleasant, the plant that adapts perfectly in Ecuador and the extract of its leaves is 200 times sweeter than sugar. Objective. The objective of this study was to carry out the economic project for the manufacture and commercialization of a taxo energy drink sweetened with stevia. Methodology. The research used a semiexperimental inductive-deductive method, variables of gender, age, level and consumption preferences, among others, were analyzed; It was executed in the Riobamba canton, to 255 people, aged 15 and over, considering various aspects. The instrument

1 Escuela Superior Politécnica de Chimborazo, Facultad de Ciencias Pecuarias, Ingeniería en Agroindustrias, Chimborazo, Riobamba, Ecuador, maria.bravo@espoch.edu.ec, https://orcid.org/ 00000003-1840-1200.

2 Escuela Superior Politécnica de Chimborazo, Facultad de Ciencias Pecuarias, Medicina Veterinaria, Chimborazo, Riobamba, Ecuador, lvelasco@espoch.edu.ec, https://orcid.org/0000-0002-4497-576.

${ }^{3}$ Universidad Estatal Amazónica, Facultad de Ciencias de la Tierra, Puyo, Ecuador, saguiar@uea.edu.ec, https://orcid.org/0000-0002-1971-7330
\end{abstract}


applied was a structured questionnaire with 12 closed questions. Results. The results showed that $86.4 \%$ of those surveyed regularly consume energy drinks from various brands in the local market. $63.6 \%$ consume it at least once a month, this shows the importance that these drinks have achieved. $36.4 \%$ of people do it for its taste and another $36.4 \%$ for quality, this demonstrated the qualities that the product should have. $86.4 \%$ of those surveyed agree to try a new flavor of this type of drink and $95.5 \%$ believe that a taxo-based energy drink would be very good and good. $72.7 \%$ of people buy their drinks in neighborhood stores, this being an ideal place to sell, the same percentage considers social networks as an optimal place to know and receive information about the product, $72.7 \%$ of those surveyed consider it appropriate to pay $\$ 1.30$ for a $250 \mathrm{ml}$ bottle. With this background we conclude that the present Economic Project for the elaboration and commercialization of a taxo energy drink sweetened with stevia is highly attractive with great potential for local entrepreneurship.

Keywords: Commercialization, market study, taxo, stevia, project.

\section{Resumen.}

Introducción. Actualmente el mercado de bebidas energizantes ha incrementado, esta investigación pretende analizar la capacidad del mercado de la ciudad de Riobamba para aceptar una bebida elaborada con un producto de la zona: el taxo, fruta que aporta vitaminas y minerales, posee un olor muy característico y agradable, la planta que se adapta a la perfección en Ecuador y el extracto de sus hojas es 200 veces más dulce que el azúcar. Objetivo. El objetivo del presente estudio fue realizar el proyecto económico para la fabricación y comercialización de una bebida energizante de taxo endulzada con estevia Metodología. La investigación uso un método inductivo-deductivo semiexperimental, se analizaron variables de género, edad, nivel y preferencias de consumo, entre otras; se ejecutó en el cantón Riobamba, a 255 personas, de 15 años en adelante, considerando varios aspectos. El instrumento aplicado fue un cuestionario estructurado con 12 preguntas cerradas. Resultados. Los resultados demostraron que el 86,4\% de los encuestados consumen regularmente bebidas energizantes de varias marcas existentes en el mercado local. El 63,6\% lo consumen como mínimo una vez al mes, esto demuestra la importancia que estas bebidas han logrado. El 36,4\% de las personas lo hacen por su sabor y otro $36,4 \%$ por la calidad, esto demostró las cualidades que debería tener el producto. El 86,4\% de los encuestados está de acuerdo en probar un nuevo sabor de este tipo de bebidas y el $95,5 \%$ creen que una bebida energizante a base de taxo sería muy buena y buena. El $72.7 \%$ de las personas adquieren sus bebidas en las tiendas de barrio, siendo este un lugar ideal de expendio, el mismo porcentaje considera a las redes sociales como un lugar óptimo para conocer y recibir información acerca del producto, el $72.7 \%$ de los encuestados considera adecuado pagar $\$ 1,30$ por una botella de $250 \mathrm{ml}$. Con estos antecedentes concluimos que el presente Proyecto Económico para la elaboración y comercialización de una bebida energizante de taxo 
endulzada con estevia es altamente atractiva con gran potencial de emprendimiento local.

Palabras claves: Comercialización, estudio de mercado, taxo, estevia, proyecto.

\section{Introducción.}

Hoy en día existe una mayor conciencia sobre los temas de salud, motivo por el cual las personas optan por productos más apegados a características naturales, sanas, nutritivas, pero al mismo tiempo a vez atrayentes al paladar. (Bravo,2019). El consumo de frutas es una elección agradable para mejorar la salud, debido a la contribución de gran cantidad tanto de vitaminas como de minerales. Ecuador es un país privilegiado ya que posee gran variedad de frutas debido a dos factores, su posición geográfica y sus microclimas, siendo estas una opción para incentivar su consumo por medio del zumo natural de frutas, mediante bebidas energizantes. (Solano, 2019)

Actualmente en Ecuador el mercado de bebidas energizantes es considerado de significativo crecimiento por la tendencia de necesidades de la sociedad por adquirir fuentes de energía necesarias durante sus actividades físicas y mentales. Las empresas continúan innovando en sus tácticas de marketing para así mantener sus marcas con un espacio en el mercado y prevenir el estado de declive. (Pacheco, 2018).

Alrededor del mercado no solo ecuatoriano sino también mundial hay un sin número de marcas respecto a las bebidas hidratantes han logrado su posicionamiento en mayor parte en la población juvenil representando así su principal segmento de mercado, la mayoría de estas bebidas hidratantes vienen en presentaciones de $250 \mathrm{ml}$, siendo distribuidas en pequeños y grandes almacenes de todo el país. (Limones, 2017).

La sociedad española de dietética y ciencias de la alimentación publica en el Libro Blanco de la Hidratación (2016), que la eliminación de agua se realiza por 3 vías; respiratoria, cutánea y renal. Razón por la cual se debe restaurar estos minerales perdidos; una opción rápido de hacerlo es a través de bebidas hipotónicas (Espinoza \& Mendóza, 2013). La solución ante la demanda existente es la creación de una microempresa dedicada a la elaboración de una bebida energética natural, ya que hoy en día el mercado no dispone de suficientes bebidas naturales saludables, por ende, se comercializan bebidas con ciertos aditivos e ingredientes químicos perjudiciales para la salud.

Por tal motivo se plantea un análisis de mercado para la creación de una microempresa, encargada de la elaboración y comercialización de una bebida energizante de taxo (Passiflora tripartita) endulzada con estevia, como una contribución al bienestar integral de los consumidores que prefieren productos orgánicos y naturales. 


\section{Cadena de valor}

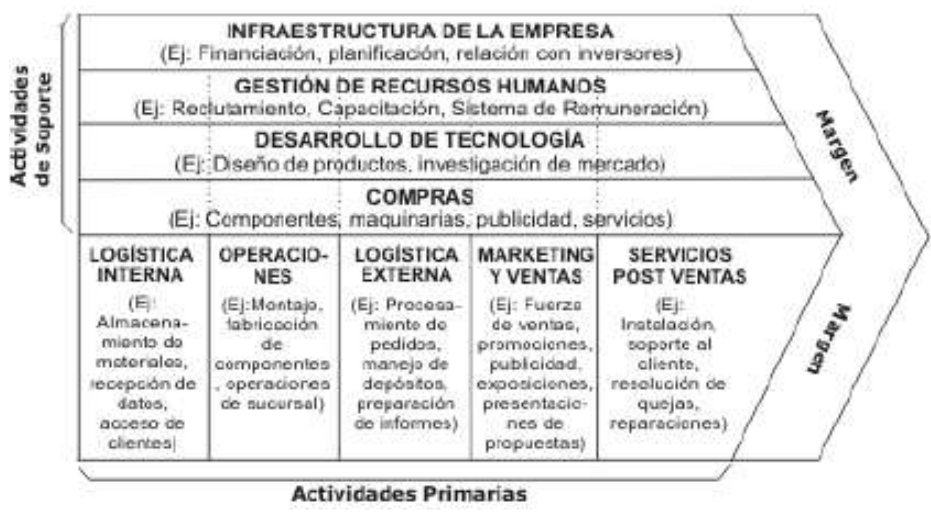

Figura 1: Cadena de valor

Fuente: Reingenia, 2011

Según Michael Porter: "La cadena de valor es un modelo teórico que gráfica y permite describir las actividades de una organización para generar valor al cliente final y a la misma empresa." La cadena de valor es una serie de actividades tanto primarias como secundarias que ayudan a crear una ventaja competitiva si las mismas son desarrolladas sinérgicamente.

Dentro de las actividades primarias se encuentran:

- Logística interna: es la provisión de insumos, materia prima, maquinaria y almacenamiento de los mismos/mismas.

- Operaciones: Es el proceso de transformación para la obtención del producto final.

- Logística externa lateral: distribución del producto a los diferentes canales de distribución.

- Marketing y ventas: son las actividades necesarias para dar a conocer el producto y generar un posicionamiento de mercado en la mente del consumidor.

- Servicio post-venta: son las actividades realizadas posteriores a la compra que controlan niveles de calidad.

- Las actividades secundarias tienen el objetivo de apoyar al cumplimiento de las actividades primarias de manera eficiente. Estas son:

- Compras: es el conjunto de actividades referentes al aprovisionamiento de la materia prima.

- Desarrollo de tecnología: son las actividades de investigación y desarrollo para la implementación de nuevos planes de negocios.

- Gestión de Recursos Humanos: son las actividades que permitirán seleccionar el personal idóneo para cada cargo. Además de realizar el plan de incentivos.

- Infraestructura de la industria: son las actividades complementarias al negocio como: contabilidad, planificación y finanzas. (Crece negocios,2 012) 
- Los canales de distribución de la industria de bebidas son: supermercados, bares, discotecas, tiendas, licorerías, supermercados, panaderías, gimnasios y gasolineras. Los canales de distribución más utilizados para adquirir bebidas alcohólicas y no alcohólicas son: mercados $40 \%$, supermercados $27 \%$ y tiendas de barrio 16\%. (IDE, 2013).

\section{El producto}

Bebida elaborada con productos de la zona como lo es el taxo, fruta que aporta muchas vitaminas y minerales, que posee un olor muy característico y agradable, además esta bebida será endulzada con estevia, planta que se adapta a la perfección en nuestro país y el extracto de sus hojas es 200 veces más dulce que el azúcar y no produce diabetes

Es por ello que la bebida energizante que se ofrecerá será elaborada a base de esta planta y presentada en una lata de aluminio de $250 \mathrm{ml}$.

\section{Estrategia de Desarrollo de Productos}

Se aplicará la estrategia de desarrollo de productos, la cual consiste en aumentar las ventas, mejorando o modificando los productos y servicios actuales. El desarrollo de productos generalmente supone grandes gastos de investigación y desarrollo. Esta estrategia se utiliza cuando una organización compite en una industria de gran crecimiento y la misma se caracteriza por los rápidos desarrollos tecnológicos. (David, 2013, p. 137)

\section{Descripción y características de la fruta}

El Taxo conocido con otros nombres como Tumbo, Parcha, Tacso, Granadilla cimarrona, Curuba, este fruto es nativo de la cordillera de los Andes, así como en la selva alta y en clima templado. Principalmente se produce en: Colombia, Venezuela, Bolivia, Perú y Nueva Zelanda. Su naturaleza de planta es ser trepadora, se enreda en distintos árboles, llegando a crecer hasta 6 metros de alto; a esta planta también le caracteriza su flor en forma de péndula y de colores atractivos en tonos rojos o violetas, por su belleza el Jardín Botánico de la ciudad de Quito la escogió como su flor representativa, su encantadora flor es familia de las orquídeas, el tallo es cilíndrico, de hojas obovadas, trilobuladas y aserradas en los márgenes. (Cuaspud, 2015).

El fruto es una baya de forma elipsoidal de aproximadamente 7 a 10 centímetros de largo, la pulpa es firme, carnosa, posee semillas de color negro pequeñas similares a la de la granadilla, su sabor es levemente dulce y ácido, el color como la mayoría de futas es verde claro cuando se está desarrollando y completamente amarillo cuando está maduro. La cosecha óptima es cuando esté pintón, esta futa se caracteriza por madurar rápidamente. El árbol de taxo produce frutos durante varios años, sus podas deben ser adecuadas de forma que favorezcan a la producción por lo menos durante ocho a diez años. (Villalobos, 2014) 


\section{Clasificación Científica.}

El taxo pertenece a la familia de las passifloras, el nombre científico: Passiflora Tripartita var. Mollissima perteneciente a la variedad que se produce en el Ecuador. El nombre de pasifloras, surgió debido a la semejanza que vieron en la flor los científicos con los instrumentos de la pasión de Cristo. Citando a Lindley, autor del Tesoro de la Botánica, mientras que Luis Cordero en su Enumeración Botánica, manifiesta que: Los tres clavos están representados por los tres pistilos; las cinco anteras de los estambres significan las cinco llagas; los rayos de gloria o la corona de espinas tienen su representación en la corona de la misma flor que posee su flore; las 10 partes o hojuelas de periantio (que parecen pétalos) denotan a los 10 apóstoles exceptuado a Pedro, quién negó a Cristo y Judas, que lo vendió. Las hojas digitadas de la planta representan las viles manos de los judíos que lo prendieron, y los zarcillos y ganchos de la misma, las cuerdas con las que ignominiosamente lo ataron. (Serrano, 2014)

La variabilidad genética en esta especie es muy alta, por lo que se encuentra una gran heterogeneidad de plantas y frutas que se diferencian en cuanto a tamaño, forma, color, resistencia, rendimiento, entre otras. (Curuba, 2014).

\section{Producción.}

La producción de esta fruta en el país es escasa, se conoce que el cultivo de taxo se produce principalmente en la provincias del Carchi, Imbabura y en mayor cantidad en la provincia de Tungurahua, que según un estudio del Instituto Nacional Autónomo de Investigaciones Agropecuarias (INIAP, 2018) el mayor porcentaje de productores que cultivan taxo se encuentran en el cantón Pelileo representando sus cultivos el 62\%; seguido de Tisaleo con el 21\%; Mocha con el $15 \%$ y Ambato con el 3\%, estos cultivos han llegado a constituirse en una fuente de recurso económico importante para los agricultores dedicados a la explotación de este cultivo, sin embargo la comercialización del taxo en estas provincias se limita a abastecer mercados populares, lo que hace aumentar los riesgos de pérdidas debido su consumo en forma fresca.

Las invetigaciones existentes confirman que el principal país que exporta esta fruta es Colombia, exporta a Europa y Estados Unidos todo el año y se registran importaciones desde Costa Rica en el mes de octubre. (Ponce, 2014)

\section{Propiedades y Aplicaciones}

Las propiedades de esta fruta es su alto aporte en proteína, calcio, fósforo, vitaminas A, B y C, consumirla ayuda a mejorar los rendimientos en las actividades diarias favoreciendo a la salud, adicional tiene un poderoso efecto sobre la musculatura de nuestro cuerpo por sus propiedades anti-espasmódicas, y analgésicas. Los extractos del género Pasiflora tienen efectos depresores sobre el sistema nervioso central y actúan como sedantes, tranquilizantes, calmantes y también contra el insomnio. También se utiliza como antiespasmódico, diaforético, hipotensor, diurético, febrífugo. La cocción 
de las hojas se emplea para el dolor de cabeza y tratar afecciones de hígado y riñones. (Angulo, 2014)

Tabla 1. Composición de la Fruta

\begin{tabular}{|l|c|}
\hline \multicolumn{1}{|c|}{ Componentes } & $\begin{array}{c}\text { Contenido de } \mathbf{1 0 0} \mathbf{g} \\
\text { de parte comestible }\end{array}$ \\
\hline Agua & $92 \%$ \\
\hline Carbohidratos & $6,30 \mathrm{~g}$ \\
\hline Fibra & $0,30 \mathrm{~g}$ \\
\hline Grasa total & $0,10 \mathrm{~g}$ \\
\hline Proteínas & $0,60 \mathrm{~g}$ \\
\hline Ácido ascórbico & $70 \mathrm{mg}$ \\
\hline Calcio & $4 \mathrm{mg}$ \\
\hline Fosforo & $20 \mathrm{mg}$ \\
\hline Hierro & $0,40 \mathrm{mg}$ \\
\hline Vitamina B3 (Niacina) & $2,5 \mathrm{mg}$ \\
\hline Vitamina B2 (Riboflavina) & $0,03 \mathrm{mg}$ \\
\hline Vitamina A & $1700 \mathrm{ug}$ \\
\hline
\end{tabular}

Fuente: Elaboración propia

\section{Aplicaciones.}

Acercándonos a la temática de la investigación de este artículo, consideramos que la fruta la principal forma de consumo es en su estado fresco, pero también tiene posibilidades de consumo en jugo, néctar, concentrados, helados y licores. Adicionalmente, se considera que una serie de productos amerita ser evaluados a partir de la fruta producida en los Andes, incluyendo: mezcla con jugos de otras frutas, sorbetes y utilización como ingrediente en la fabricación de crema glasé y artículos de pastelería y confitería. (Romero, 2013).

La estrategia de producto expone referentes esenciales para lograr que la bebida se desarrolle adecuadamente; tales como ciclo de vida, portafolio y marca; a través de la promoción se pretende desarrollar una estrategia efectiva para llamar la atención del consumidor final, de esta forma se motiva a probar y seguir consumiendo el nuevo producto; el precio es establecido a través de una inicial investigación de mercado con el cálculo aproximado de la demanda, los costos asociados al producto y la tercerización de los mismos, llegando así a una base de la cual se debe partir; y finalmente lo que respecta a la plaza se encuentran cuatro principales canales de distribución, estos permiten satisfacer las necesidades de los mismos, encontrándose en el lugar donde exactamente estas les surgen. (Gomez \& López, 2013). 


\section{Metodología.}

\section{Descripción y contexto de los participantes}

La presente investigación se realizó mediante un método inductivo-deductivo, semi experimental, (Abreu, 2014), luego de obtener los datos estos fueron sometidos a un análisis descriptivo, trabajando con medidas de tendencia central y dispersión; se analizaron variables como: Género, edad, nivel de consumo, preferencias y tendencias de consumo, entre otras.

La recopilación de información se realizó después de una delimitación geográfica, psicográfica y demográfica, determinando la investigación de mercado en la Provincia de Chimborazo, cantón Riobamba en las 4 parroquias urbanas; la encuesta se aplicó a 255 personas, de 15 años en adelante, tomando en cuenta muchos aspectos como: calidad, disponibilidad, precio, representación y distribución.

\section{Instrumentos}

El instrumento aplicado fue un cuestionario, mismo que fue estructurado con 12 preguntas de tipo cerradas y con escala de Likert, que constituye uno de los instrumentos más utilizado en los estudios de mercado para obtener datos de calidad. (Matas, 2018).

\section{Procedimiento}

El método que se aplicó para la recopilación de datos fue la información fue online, a través de la herramienta Google forms, las encuestas fueron enviadas mediante las redes sociales como: Facebook, Instagram y WhatsApp

\section{Resultados.}

En la presente investigación participaron 255 personas, de las cuales 151 fueron hombres con el 59,1\% y 104 fueron hombre con el 40.9\%, siendo este el universo en el cual trabajamos la presente investigación.

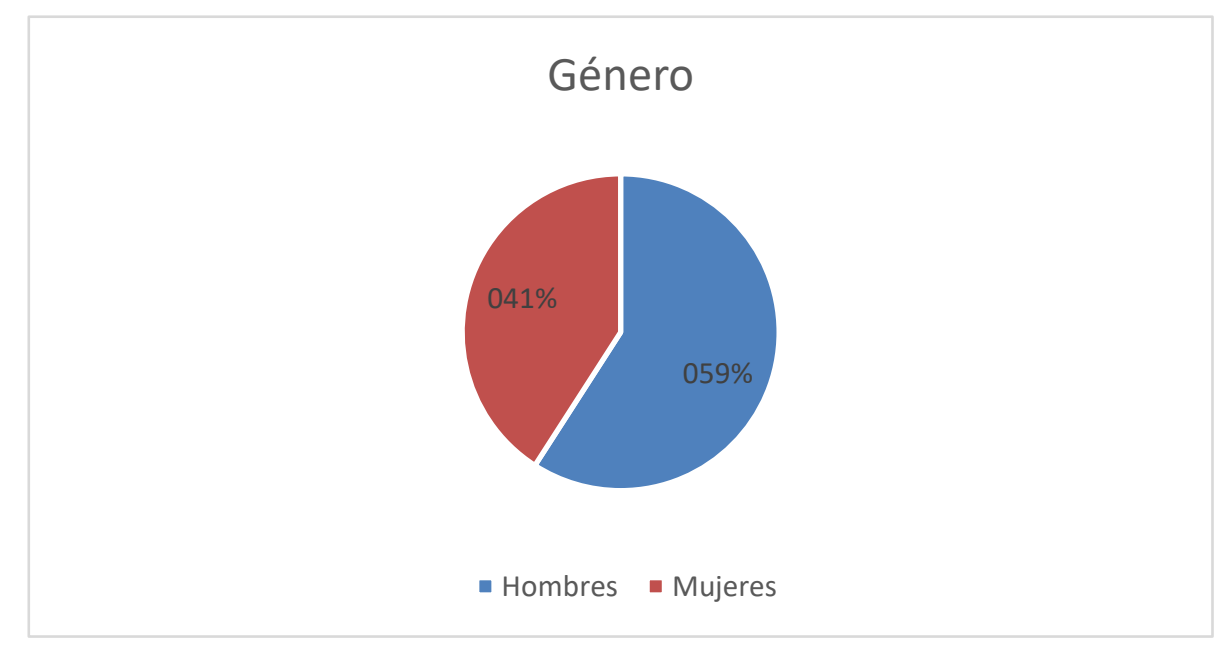

Figura 2. Género de los encuestados 
Participaron 232 personas con una edad de entre 15 a 25 años, lo cual representa el 90.9\% de los participantes, también 23 personas con una edad de entre 26 a 45 años, lo que representa el 9.9\%. En la investigación 220 personas reportaron que si consumen bebidas energizantes, lo cual representa el $86.4 \%$, y 35 personas reportaron que no consumen bebidas energizantes, lo cual representa el $13.6 \%$.

\section{Consumo de bebidas energizantes}

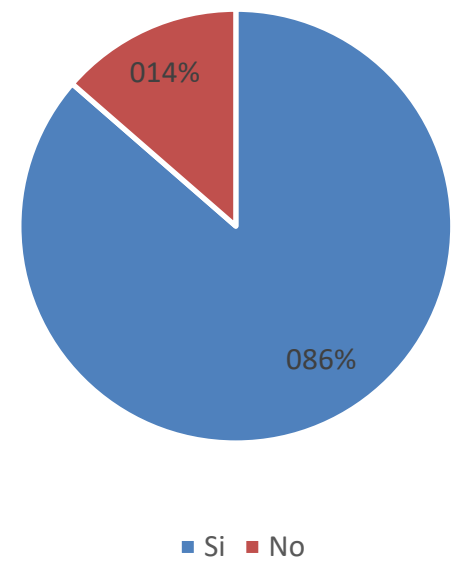

Figura 3. Consume usted bebidas energizantes

El análisis de la frecuencia en el consumo de bebidas energizantes nos indica que 162 personas la consumen de forma mensual, lo cual representa el $63.6 \%, 35$ personas la consumen cada 3 meses, lo cual representa un 13.6\%, 12 personas la consumen cada 4 meses lo que representa el $4.6 \%$ y 46 personas la consumen cada 6 meses lo que representa un $18.2 \%$.

\section{Frecuencia de Consumo}

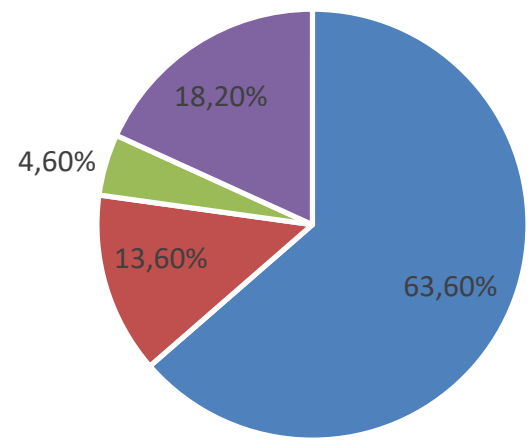

- Cada mes - Cada 3 meses - Cada 4 meses - Cada 6 meses

Figura 4. Frecuencia de consumo de bebidas energizantes. 
En cuanto a la investigación de mercado específicamente con lo que respecta a las marcas de las bebidas energizantes que más se consumen, nos encontramos que 93 personas prefieren Vive 100, lo cual representa el 36.4\%, 70 personas consumen Powerade lo que representa el $27.3 \%, 58$ personas consumen $220 \mathrm{~V}$ que representa el $22.7 \%, 26$ personas consumen Volt que representa el 10.1\%, 7 personas consumen Speed que representa el 3\%, 1 persona reporta consumir otras marcas, lo cual representa el $0.5 \%$.

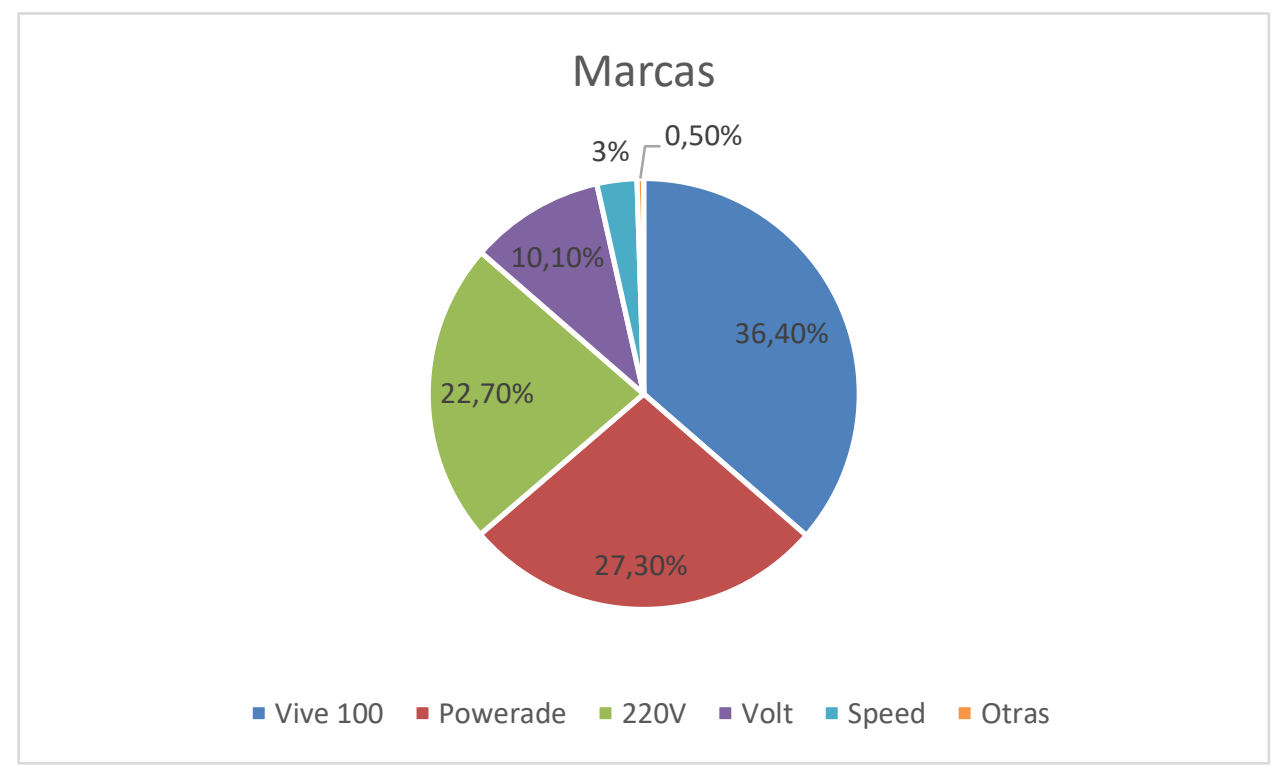

Figura 5. Marcas de energizantes consumidas con mayor frecuencia.

En lo que respecta a la tendencia de consumo las razones de los consumidores y sus preferencias es que 93 personas lo hacen por su calidad lo que representa el 36.4\%, 93 personas lo hacen por el sabor lo cual representa el $36.4 \%, 58$ personas lo hacen por su precio lo que representa el $22.7 \%, 8$ personas lo hacen por su presentación que representa el $3 \%$ y 3 personas lo hacen por otras razones lo que representa el $1.5 \%$.

\section{Razones de preferencia}

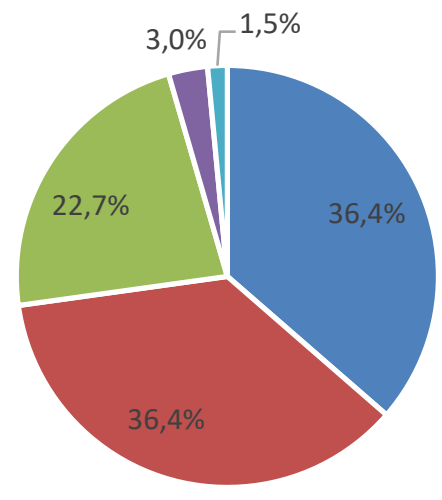

$$
\text { - Calidad - Sabor - Precio - Presentación - Otro }
$$

Figura 6. Razones de preferencia de compra de una marca de energizante. 
Continuando con las preferencias de consumo e investigando sobre el sabor de preferencia de los consumidores, 70 personas reportaron el sabor de limón lo cual representa el $27.3 \%, 58$ personas dijeron manzana que representa el $22.7 \%, 56$ personas dijeron uva que representa el 22\%, 38 personas dijeron Sandía que representa $15 \%$ y 33 personas reportaron otro sabor que representa el $13 \%$.

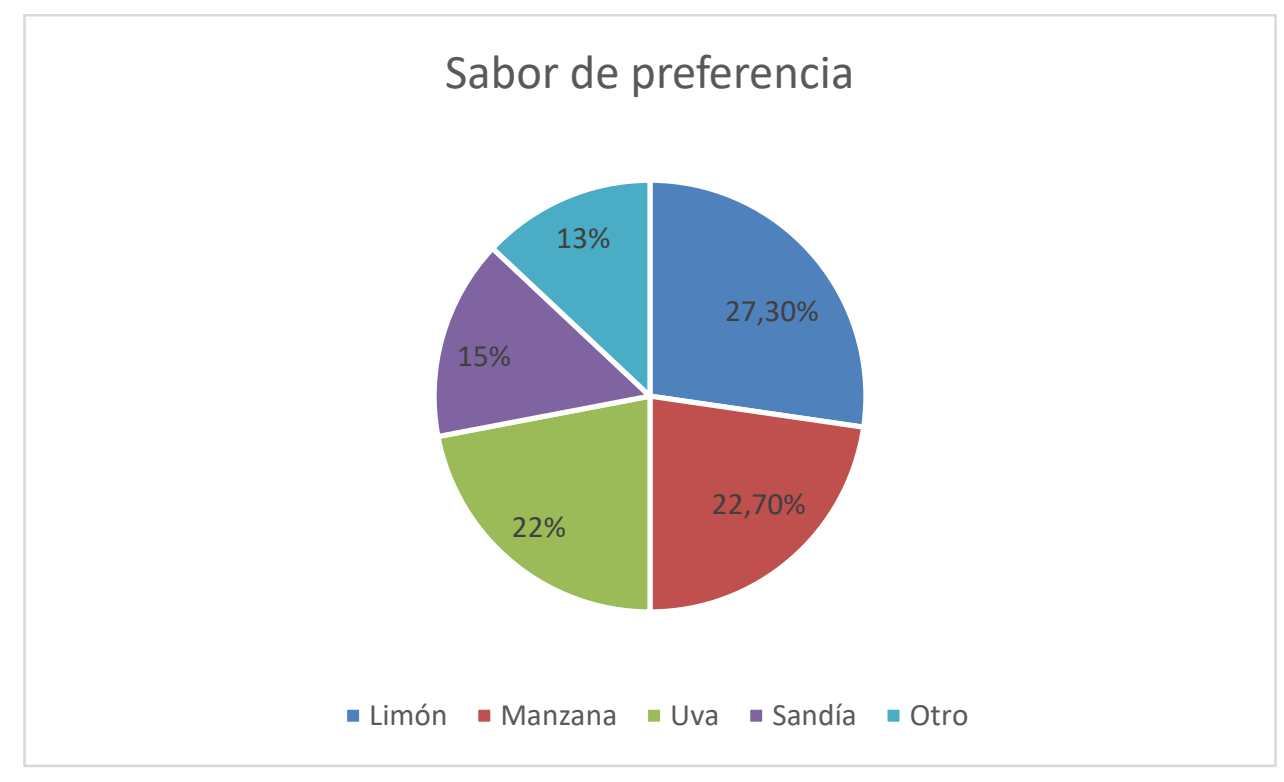

Figura 7. Sabor preferido en una marca de energizante.

Investigando específicamente el mercado se planteó la pregunta sobre si probarían otro sabor, 220 personas reportaron que si estarían interesadas en hacerlo, lo cual representa el $86.4 \%$, y 35 personas reportaron que no están interesados, lo cual representa el $13.6 \%$, dándonos apertura en el mercado para el producto planificado.

\section{Probaría un nuevo sabor}

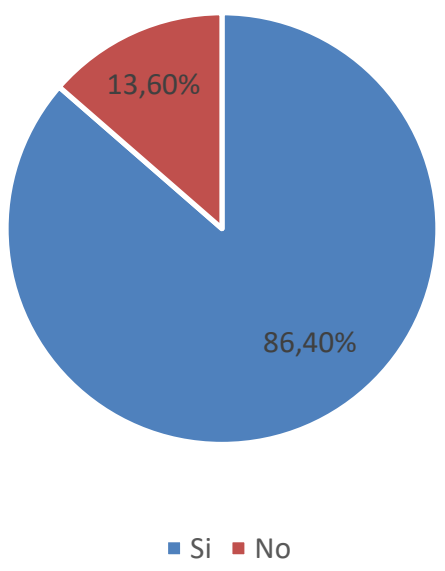

Figura 8. Propuesta de nuevo sabor de energizante 
Con respecto a la introducción al mercado local de una nueva bebida energizante a base de taxo, 93 personas creen que sería muy buena lo que representa el 36.4\%, 150 personas consideran que sería buena lo que representa un $59.1 \%, 8$ personas creen que sería regular lo que representa el $3 \%$ y 4 personas creen que sería mala lo que representa el $1.5 \%$.

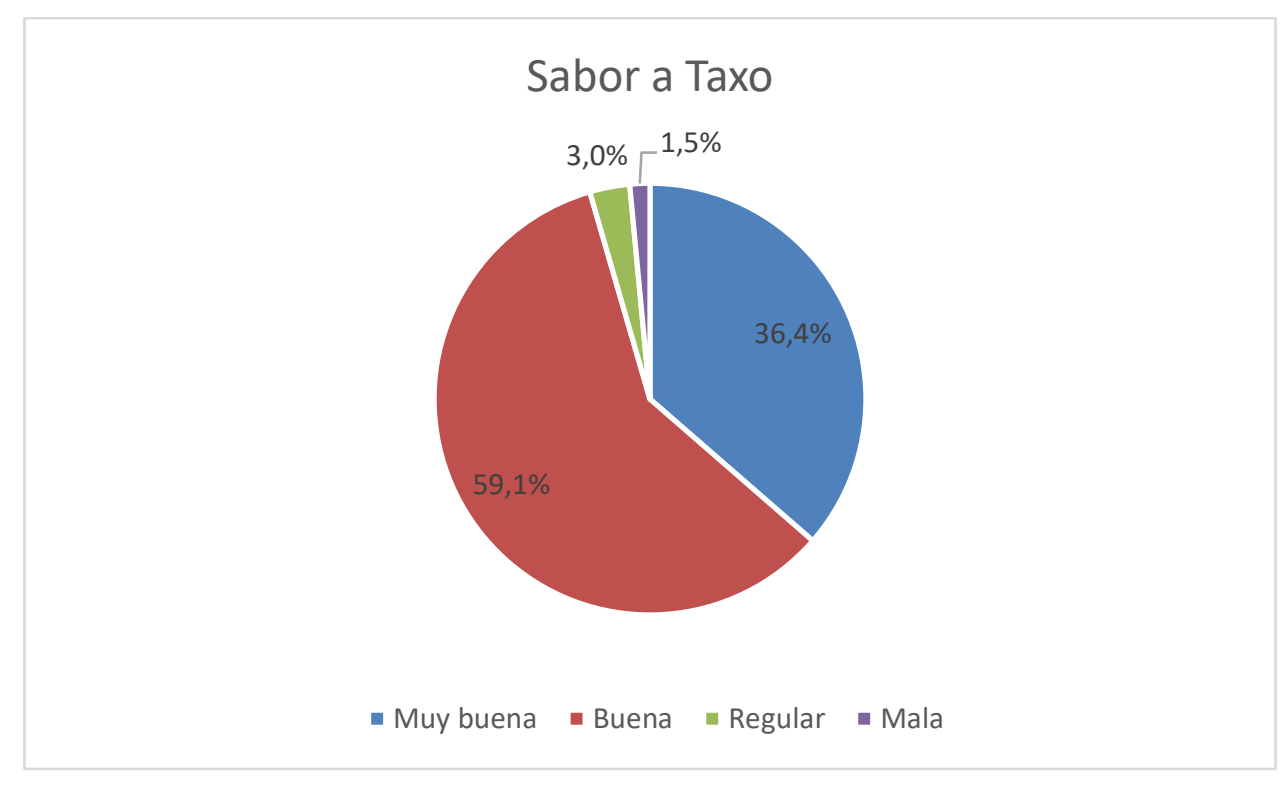

Figura 9. Aceptación de bebida energizante a base de taxo.

En esta investigación de mercado también se indagó si la población objeto de estudio adquirirían esta nueva bebida, 104 personas respondieron que sería muy probable lo que representa el 40.9\%, 128 personas respondieron que probablemente lo que representa el 50\%, 23 personas respondieron que poco probable lo que representa el $9.1 \%$ y nadie respondió que nunca probable.

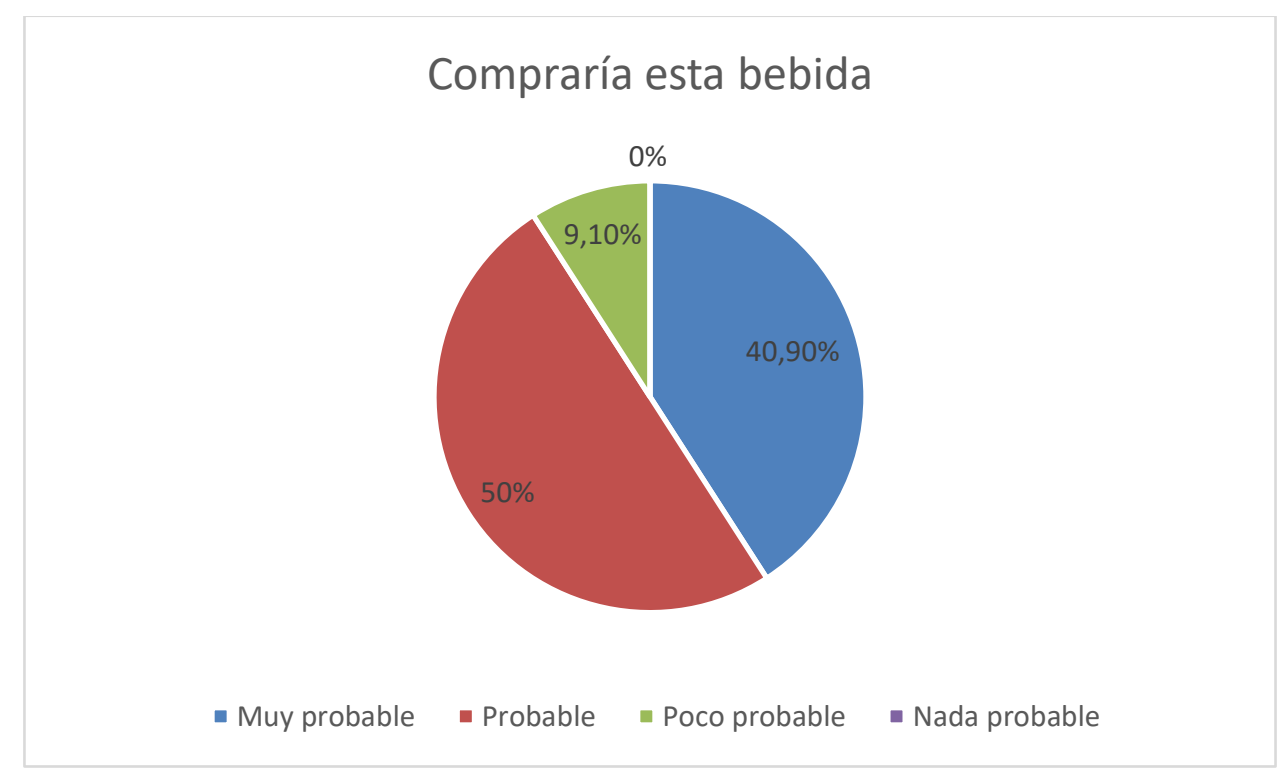

Figura 10. Adquisición de energizante a base de taxo. 
Esta investigación de mercado amplía su campo hasta la comercialización, respecto al lugar preferido de compra de las bebidas energizantes, 185 personas respondieron que, en una tienda de barrio, lo que representa en 72.7\%, 58 personas lo hacen en los Supermercados lo que representa el $22.7 \%$ y 12 personas lo hacen en el Mercado lo que representa el $4.6 \%$.

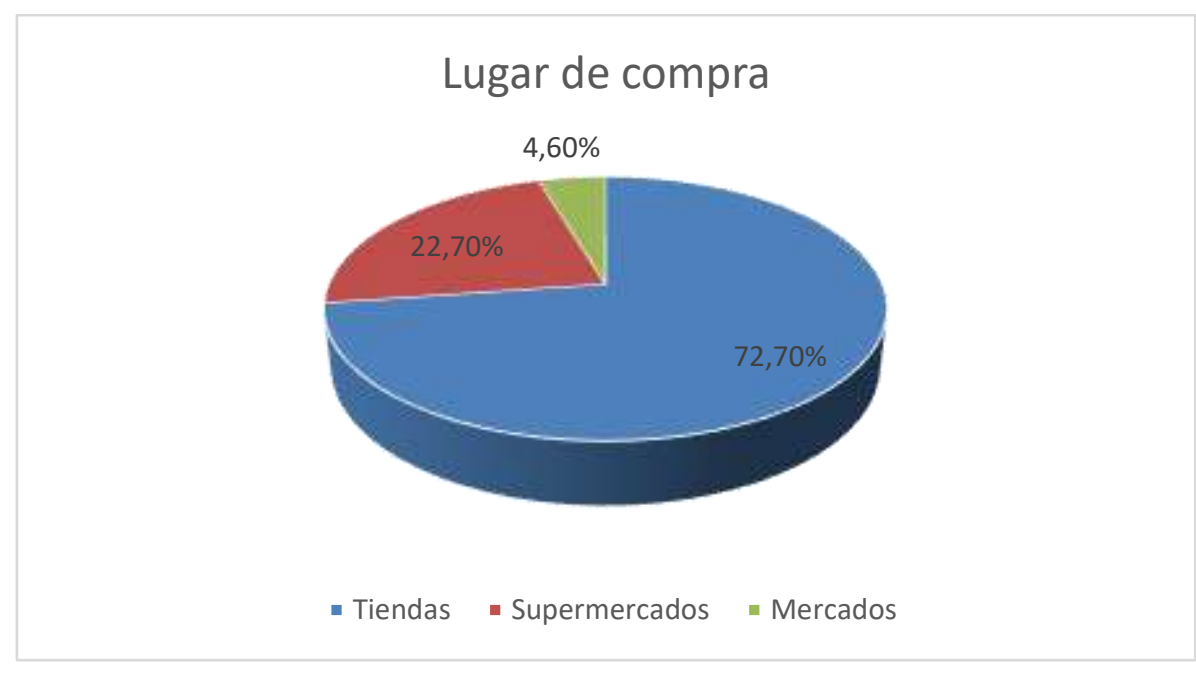

Figura 11. Lugar de compra de las bebidas energizantes.

Otro punto sumamente importante en esta investigación y que realmente impacta en la población objeto de estudio es el precio, el valor propuesto para su adquisición es de $\$ 1,30$ por una botella de $250 \mathrm{ml}, 47$ personas respondieron que el valor es caro lo que representa el $18.2 \%, 185$ personas consideran que el precio es normal lo que representa el $72.7 \%$ y 23 personas consideran que el precio es barato, lo que representa el $9.1 \%$.

\section{Precio de la bebida}

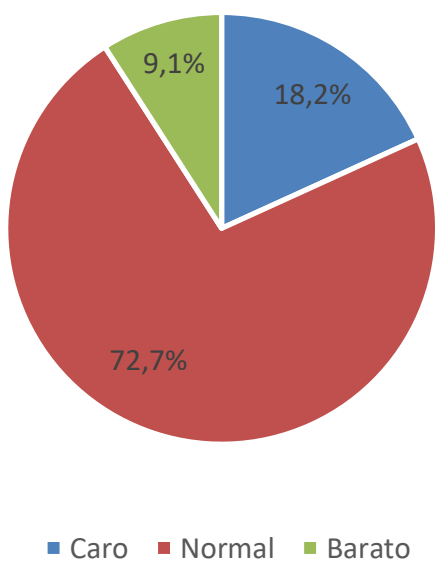

Figura 12. Precio por botella de $250 \mathrm{ml}$ de energizante a base de taxo.

Finalmente, la investigación concluye con la publicidad, la pregunta sobre por cual medio de comunicación le gustaría recibir información sobre el producto, 185 personas 
contestaron que por Redes Sociales lo que representa el 72.7\%, 58 personas respondieron que por Televisión lo que representa el $22.7 \%$ y 12 personas contestaron que por Radio lo que representa el $4.6 \%$.

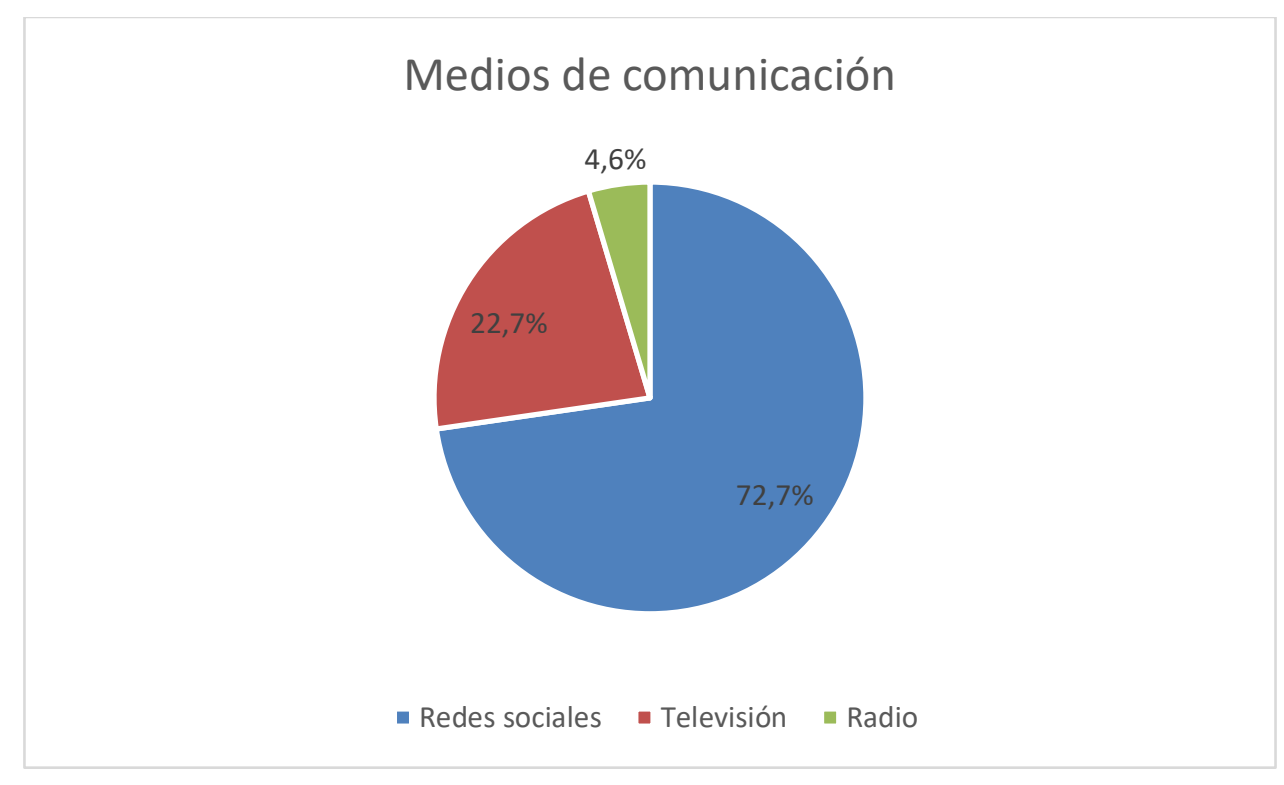

Figura 13. Medio de Comunicación de preferencia.

A nivel mundial y de Ecuador la producción es necesaria para crear bienes económicos haciendo posible la satisfacción de necesidades, siempre y cuando se incorpore una utilidad (Parra, 2008). El taxo una fruta poco explotada y cultivada en otros países, pero si muy considerando por su particular sabor y alto valor nutricional. (Cetre, 2015).

Para la producción de una bebida energizante a base de taxo y su comercialización, considerando el valor de la producción que mide el importe de los bienes producidos, la comercialización que en marketing que es el proceso de planificación y control del producto y asegurar el momento, precio y cantidad necesarios para garantizar su venta (kotler, 2007), después de la investigación de mercado realizada y en concordancia con varios autores citados en esta investigación, con los resultados obtenidos demostramos que es posible la creación de una microempresa encargada de la elaboración y comercialización de una bebida energizante de taxo con el plus de ser endulzada con estevia, cumpliendo el objetivo de esta investigación, el demostrar que es posible realizar la bebida antes mencionada.

\section{Conclusiones.}

- El presente proyecto económico para la elaboración y comercialización de una bebida energizante de taxo endulzada con estevia es altamente atractiva, pues un $86 \%$ de las personas consumen con frecuencia estos productos y los resultados del estudio de mercados que reflejarán la fuerte intención de adquirir este producto y establecer una gran oportunidad de emprendimiento dentro de un plan de negocios. 
- El mercado existente, necesita y requiere esta clase de productos nuevos, y lo mejor, estarían dispuestos a pagar por nuestro producto, para ello se aplica técnicas y estrategias de publicidad y promoción que sean atractivas para los potenciales consumidores de acuerdo a nuestra segmentación de mercado y de esta manera posicionar nuestra marca.

- Los recursos que se necesita principalmente para el desarrollo y funcionamiento del negocio es la materia prima la cual es basta y muy accesible, además para el desarrollo de tácticas de marketing es importante identificar las fortalezas y debilidades, considerar el actual entorno competitivo presente en las bebidas energizantes en conjunto con las necesidades que posee el consumidor, los factores que influyen al momento de obtener una bebida energizante y las preferencias existentes hacia una marca en particular, debido a ello la bebida energizante "Taxoliff" podría generar ventas a través de la fidelización de los clientes a la vez de dar un valor agregado a la materia prima inicial

\section{Referencias bibliográficas.}

Abreu, Jose., (2014). El método de la Investigación. Revista electrónica, Daena. Recuperado en 20 de enero de 2021, de http://www.scielo.org.mx/scielo.php?script=sci_arttext\&pid=S1607$40412018000100038 \& \operatorname{lng}=$ es\&tlng=es.

Angulo, Rafael. Frutales exóticos de Clima Frío, [en línea], Bayer CropScience S.A., 2003. [fecha de consulta: 22 de agosto del 2020]. Disponible en: http://www.fao.org/inpho_archive/content/documents/vlibrary/ae620s/pfrescos/c uruba.htm.

Bravo, María., \& Miranda, María., (2020). Análisis teórico nutritivo de las galletas de remolacha (Beta Vulgaris) y su tendencia de consumo y comercialización. Congreso SEPPEA, ESPOCH.

Cabrera, F. (2020)., LICENCIA URBANÍSTICA DE URBANIZACIÓN. Obtenido de http://www.gadmriobamba.gob.ec/formularios_tramites/documentos/TRAMELU-016.pdf

Cetre, C. (2015)., estudio de factibilidad para la creación de una microempresa de comercialización de borojó orgánico con calidad de exportación de acuerdo con las exigencias del mercado alemán en la comunidad awá, cantón san lorenzo, provincia de Esmeraldas, PUCE. Obtenido de https://repositorio.pucese.edu.ec/bitstream/123456789/361/1/CETRE\%20PERE A\%20CYNTHIA.pdf

Crecenegocios. (2012). Cadena de valor. Recuperado el 27 de diciembre del 2020. URL: http://www.crecenegocios.com/cadena-de-valor. 
Cuaspud, Yola., (2015). Elaboración de bebidas naturales a partir de taxo (passiflora tripartita var. Mollissima) y piña (ananas comosus) enriquecidas con lactosuero, Quito. Disponible en: http://www.dspace.uce.edu.ec/bitstream/25000/4771/1/TUCE-0017-129.pdf

Curuba, Curubas, Taxo, Tumbo, Parcha, Tacso, Granadilla cimarrona. Infojardín [en línea], 2006, [fecha de consulta: 22 de agosto del 2020]. Disponible en: http://articulos.infojardin.com/Frutales/fichas/curubas-taxo-tumbo-parchatacsopassiflora-mollisima.htm

David, F. (2013). Conceptos de administración estratégica. México: Pearson Prentice Hall.

Frutasyverdurascastellon. (2017). frutasyverdurascastellon.com. Obtenido de https://www.frutasyverdurascastellon.com/propiedades-naranjas-castello/

Espinoza, J., \& Mendóza, C. (2013). Factibilidad para la introducción de agua hidratante al mercado de la provincia de Manabí. Calceta. Manabí: Escuela Superior Politécnica Agropecuaria de Manabí.

Glanville, A. (2016). Oferta y Demanda. Madrid: Books. p. 224.

Gomez, D., \& López, J. P. (2013). PLAN ESTRATÉGICO PARA EL LANZAMIENTO DE BEBIDAS ENERGIZANTES EN MEDELLÍN. Obtenido de https://repository.eia.edu.co/bitstream/handle/11190/769/GomezDaniel_2013_Pl anEstrategicoLanzamiento.pdf;jsessionid=E974688995B43B512C1BF1F022895 9F4? sequence $=7$

IDE. (2013). El consumo en el Ecuador: indicadores exclusivos. URL:http://investiga.ide.edu.ec/index. Recuperado el 2 de Octubre del 2020.

Instituto Nacional de Estadística y Censos del Ecuador . (2015). Riobamba · Población. Obtenido de http://poblacion.population.city/ecuador/riobamba/

Instituto Nacional de Investigaciones Agropecuarias. (2018). Tunhuragua. Obtenido de: https://paisenvivo.com/iniap-analiz-la-produccin-de-taxo-en-tungurahua/

:Kotler, P., (2007). Dirección de Mercadotecnia, Análisis, planeación, implementación y control. México: Mc. Graw-Hill.

Limones, C. (14 de Septiembre de 2017). Plan de Marketing para la introducción de una Bebida Hidratante a base de Lactosuero y enriquecida con Vitaminas en la ciudad de Guayaquil. Obtenido de http://repositorio.ucsg.edu.ec/bitstream/3317/8793/1/T-UCSG-PRE-ESP-MDCM-138.pdf

Matas, Antonio. (2018). Diseño del formato de escalas tipo Likert: un estado de la cuestión. Revista electrónica de investigación educativa,20(1), 38-47. 
Recuperado en 20 de enero de 2021, de http://www.scielo.org.mx/scielo.php?script=sci_arttext\&pid=S1607$40412018000100038 \& \operatorname{lng}=$ es\&tlng=es.

MInisterio de Cultura y Patrimonio. (2013). Obtenido de https://www.culturaypatrimonio.gob.ec/wpcontent/uploads/downloads/2013/12/5-PATROMINO-1.pdf

Pacheco, J. (Septiembre de 2018). Diseño de estrategias de marketing para la marca de energizante "Volt" en la ciudad de Guayaquil. Obtenido de http://repositorio.ug.edu.ec/bitstream/redug/35563/1/TESIS\%20MARCA\%20V OLT\%20-\%20JOHNNY\%20PACHECO.pdf

Parra, Ernesto. (2008). Gestión de la Producción . México: MSC

Pérez, S. (2013). Obtenido de https://repositorio.uide.edu.ec/bitstream/37000/357/1/TUIDE-0336.pdf

Ponce, Javier. Estudio, análisis y propuesta gastronómica del taxo (Passiflora mollisima) [en línea]. Trabajo de grado. Administrador Gastronómico. Universidad Tecnológica Equinoccial, Facultad de Turismo y Preservación Ambiental, Hotelería y Gastronomía. Escuela de Gastronomía. Quito 2009. [fecha de consulta: 21 de agosto del 2020]. Disponible en: http://repositorio.ute.edu.ec/bitstream/123456789/11382/1/37335_1.pdf

Reingenia. (2011). La Cadena de Valor: Como optimizar el valor al cliente. URL: http://www.re.-ingenio.com/bolg/2011/01-la-cadena-de-valorcomooprtimizar-el-valor-al-cliente. Recuperado el 17 de Enero del 2013.

Romero, Carlos., (2013). Elaboración de mercados y mistelas con especies vegetales disponibles en la provincia del Azuay. Disponible en: https://dspace.ucuenca.edu.ec/bitstream/123456789/3697/1/Tesis.pdf

Serrano, Vladimir. El taxo, denominada la flor de Quito. El Comercio, [en línea], 29 de octubre del 2010, [fecha de consulta: 22 de agosto del 2020]. Disponible en: http://www.elcomercio.com/actualidad/quito/taxo-denominada-florquito.html.

Solano, Marcos., (2019). Plan de negocios para la elaboración de jugos naturales a base de verduras en la ciudad de Cuenca. UCSG. Disponible en: http://repositorio.ucsg.edu.ec/bitstream/3317/13315/1/T-UCSG-POS-MAE246.pdf

Villalobos, Byron. Las propiedades de la tuna y el taxo. [en línea], 4 de junio del 2012, [fecha de consulta: 22 de agosto del 2020]. Disponible en: http://taxoytuna.blogspot.com/2012/06/taxo.html 
Zamora Mora, V., Soares, P., Echeverria, C., Hernández , R., \& Mijangos, C. (2015). Composite chitosan/Agarose ferrogels for potential applications in magnetic hyperethermia. Gels., 1, 69-80.

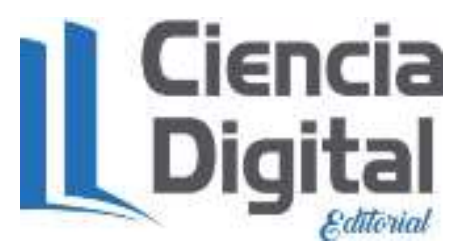




\section{PARA CITAR EL ARTÍCULO INDEXADO.}

Bravo Avalos, M. B., Velasco Matveev, L. A., \& Aguiar Novillo, S. N. (2021). Proyecto económico para la fabricación y comercialización de una bebida energizante de taxo endulzada con estevia. ConcienciaDigital, 4(3.1), 405-423. https://doi.org/10.33262/concienciadigital.v4i3.1.1854

\section{Ciencia \\ Digital \\ Editorial}

El artículo que se publica es de exclusiva responsabilidad de los autores y no necesariamente reflejan el pensamiento de la Revista Conciencia Digital.

El artículo queda en propiedad de la revista y, por tanto, su publicación parcial y/o total en otro medio tiene que ser autorizado por el director de la Revista Conciencia Digital.

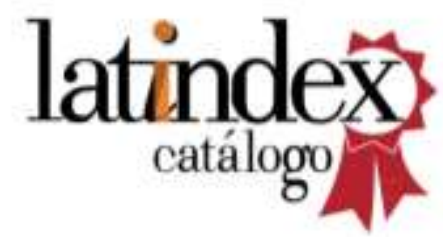

